Pacific Journal of Mathematics

DUAL GENERALIZATIONS OF THE ARTINIAN AND 


\title{
DUAL GENERALIZATIONS OF THE ARTINIAN AND NOETHERIAN CONDITIONS
}

\author{
ROBERT C. SHOCK
}

An Artinian module can be characterized in terms of certain properties of its factor modules. A module $M$ is Artinian if and only if the following two conditions hold for $M$ :

(I) Every nonzero factor module of $M$ contains a minimal submodule. ed.

(A) The socle of every factor module of $M$ is finitely generat-

The dual to the factor module is the submodule. We state the dual of (I): module.

(II) Every nonzero submodule of $M$ contains a maximal sub-

We call a module with property (II) a Max module and one with property (I) a Min module. Every Noetherian module is a Max module but not conversely. This paper investigates these generalizations of the Artinian and Noetherian conditions and the relationships among them.

Throughout this paper $M$ denotes a right module over an arbitrary (associative) ring which need not have an identity. We denote the socle of $M$ (the sum of the minimal submodules, [1]) by $s(M)$ and its dual notion, the radical of $M$ (the intersection of the maximal submodules, [6]), by rad $M$. We define $s(M)=(0)$ whenever $M$ contains no nonzero minimal submodules. We call a submodule of $M$ essential if it has nonzero intersection with every nonzero submodule of $M$. A submodule $A$ of $M$ is said to be small in $M$ in case $A+B=M$ implies that $B=M$ where $B$ is a submodule of $M$. The sum of all the small submodules of $M$ is the radical of $M$ (Eckmann [9, p. 58]) and the intersection of the essential submodules of $M$ is the socle of $M$ (Sandomierski-Kasch, [9, p. 62]). It is straightforward to see that factor modules have finitely generated socles if and only if factor modules are (Goldie) finite dimensional.

We state a major result. A module $M$ is Noetherian if and only if (A) holds and factor modules are Max modules. This result exhibits several dual notions. Assume that (A) holds for a module $M$. The following conditions are equivalent: (a) $M$ is Noetherian (Artinian), (b) every factor module of $M$ is a Max module (Min module), (c) in a factor module the radical of a submodule is small (in a factor module the socle of a submodule is essential), and (d) in a factor module the radical of a submodule 
is finitely generated (in a factor module the socle of a submodule is finitely embedded [7]).

R. P. Kurshan has shown that a ring $R$ with 1 is Noetherian if and only if $R$ (as a module) has property (A) and a T.C. condition [7, p. 379]. We show that the T.C. condition implies the Max property but not conversely (Proposition 3.10 and Example 3.13). Also, K. Goodearl has pointed out that the Max property is not homomorphic invariant; the ring of integers $Z$ localized at the powers of 2 as a $Z$-module is a Max module $M$ but $M / Z$ is isomorphic to $Z_{2} \infty$ and is not a Max module.

We now present some elementary properties of the Max module and Min module via pre-torsion theories relative to the class of simple modules.

2. Preliminaries and pre-torsion theories. Let $M$ be a Min module. If the socle of $M$ were not essential then for appropriate nonzero submodule $A$ the direct sum $s(M)+A$ would be an essential submodule of $M$. Hence, $A+s(M) / s(M)$ is essential in $M / s(M)$ and thus $M / s(M)$ contains no minimal submodule, a contradiction. From this argument we conclude that $M$ is a Min module if and only if every nonzero factor module has an essential socle.

We now "index" with respect to the socle. For a module we define for each ordinal $\alpha$ a submodule $S(\alpha)$ as follows: (i) $S(0)=0$ (ii) if $\beta=\alpha+1$ is not a limit ordinal assign $S(\beta)$ to the module such that $S(\beta) / S(\alpha)$ is the socle of $M / S(\alpha)$ (iii) if $\beta=\alpha+1$ is a limit ordinal then set $S(\beta)=\Sigma S(\sigma)$ $($ all $\sigma<\beta)$. There is an ordinal $\gamma$ such that $S(\gamma+1)=S(\gamma)$. We set $S(\gamma)=$ $S$-soc $M$ ( successive socles of $M$ ).

Proposition 2.1. For a module $M$, the following statements are equivalent:

(1) The module $M$ is a Min module.

(2) Factor modules of $M$ have essential socles.

(3) The $S$-soc $M=M$.

(4) There exists a well-ordered increasing sequence $M(0), \ldots, M(x), \ldots$, $M(\gamma)=M$ where proper containment $M(x) \subset M(x+1)$ implies that $M(x+$ $1) / M(x)$ is a minimal submodule.

Proofs. These implications are clear: (1) implies (2), (2) implies (3), (3) implies (4), (4) implies (1).

The dual to the above procedure is to take successive radicals. For a module $M$ we define for each ordinal $\alpha$ a submodule $R(\alpha)$ as follows: (i) $R(0)=M$ (ii) if $\beta=\alpha+1$ is not a limit ordinal then $R(\beta)$ is defined to be 
the $\operatorname{rad} R(\alpha)($ iii) if $\beta=\alpha+1$ is a limit ordinal then $R(\beta)=\cap R(\sigma)$ (all $\sigma<$ $\beta)$. There is an ordinal $\gamma$ such that $R(\gamma+1)=R(\gamma)$ and we set $R(\gamma)=$ $R$-rad $M$ (Radical of the radical). We state the dual of Proposition 2.1.

Proposition 2.2. For a module $M$ the following statements are equivalent:

(1) The module $M$ is a Max module.

(2) Nonzero submodules of $M$ have small radicals.

(3) The $R-\operatorname{rad} M=(0)$.

(4) There exists a well-ordered decreasing sequence $M(0)=M, \ldots, M(x)$, $\ldots, M(r)=(0)$ where proper containment $M(x) \supset M(x+1)$ implies that $M(x+1)$ is a maximal submodule of $M(x)$.

Proof. If the radical of a nonzero submodule $K$ is small, then $K \neq$ $\operatorname{rad} K$ and $K$ contains a maximal submodule. The implications are clear: (1) implies (2), (2) implies (3), (3) implies (4) and (4) implies (1).

Throughout we adopt the torsion theory terminology of [8]. Let $\mathscr{A}$ be the class of simple $R$-modules. Equate the following:

$\mathscr{A}^{r}=\{C: \operatorname{Hom}(A, C)=0$ for all $A$ in $\mathscr{A}\}$

$\mathscr{A}^{l}=\{B: \operatorname{Hom}(B, A)=0$ for all $A$ in $\mathscr{A}\}$

$\mathscr{A}^{r l}=\left\{K: \operatorname{Hom}(K, C)=0\right.$ for all $C$ in $\left.\mathscr{A}^{r}\right\}$

$\mathscr{A}^{l r}=\left\{N: \operatorname{Hom}(B, N)=0\right.$ for all $B$ in $\left.\mathscr{A}^{l}\right\}$

Then $\left(\mathscr{A}^{l}, \mathscr{A}^{l r}\right)$ and $\left(\mathscr{A}^{r l}, \mathscr{A}^{r}\right)$ are pre-torsion theories. Immediately, $\mathscr{A}^{r}$ is precisely the class of modules with no nonzero minimal submodules; equivalently, $M$ is in $\mathscr{A}^{r}$ provided that $S$-soc $M=0$. Also, $\mathscr{A}^{l}$ is precisely the class of modules with no maximal submodules, that is, $M$ is in $\mathscr{A}^{l}$ if and only if the $R-\operatorname{rad} M=M$.

Proposition 2.3. (1) $\mathscr{A}^{\text {rl }}$ is the class of Min modules and $\mathscr{A}^{\text {lr }}$ is the class of Max modules.

(2) The corresponding idempotent radical for $\left(\mathscr{A}^{r l}, \mathscr{A}^{r}\right)$ is the S-soc of a module. The corresponding idempotent radical for $\left(\mathscr{A}^{l}, \mathscr{A}^{\text {rr }}\right)$ is the $R$-rad of a module.

(3) The class of Min modules is closed under isomorphic images, submodules, group extensions and direct products.

(4) The class of Max modules is closed under isomorphic images, factor modules, group extensions and direct sums.

Proof. Statements (1) and (2) are clear. Statements (3) and (4) follow from properties of pre-torsion theories (Proposition 0.1 of [8]). 
The pre-torsion theory $\left(\mathscr{A}^{r l}, \mathscr{A}^{r}\right)$ is mentioned in [2].

3. The Artinian and Noetherian condition. In this section our goal is to prove this basic result. Let $M$ be an infinitely generated module. Then some factor module is not a Max module or some factor module has an infinitely generated socle with a zero radical. We first look for relationships between Min modules and Artinian modules.

Proposition 3.1. A module is Artinian if and only if it is a Min module and factor modules have finitely generated socles.

Proof. Assume that $M$ is a Min module and factor modules have finitely generated socles. Suppose that $A_{1} \supset A_{2} \supset \ldots$ is a strictly decreasing sequence of nonzero submodules of $M$ and $\cap A_{i}(i \geq 1)=0$. Clearly, $s(M)$ is essential and forces $A_{i} \cap s(M) \neq(0)$ for all $i \geq 1$. Also, $s(M)$ is finitely generated; equivalently, $s(M)$ has the descending chain condition. Therefore, for appropriate $n, s(M) \cap A_{n}=s(M) \cap A_{n+j} \neq 0$ for all $j \geq 1$, a contradiction of $\cap A_{i}(i \geq 1)=0$. For an arbitrary decreasing sequence $A_{1} \supset A_{2}, \ldots$ we apply the above argument to $M / \cap A_{i}$. The other implication is clear. This completes the proof.

Proposition 3.2. An Artinian module $M$ is Noetherian if and only if $M$ is a Max module.

Proof. Suppose that $M$ is Artinian but not Noetherian. Let $A$ be a minimal submodule which is not Noetherian. By hypothesis $A$ contains a maximal submodule $A^{\prime}$. The minimality of $A$ forces $A^{\prime}$ to be Noetherian and hence $A$ is Noetherian, a contradiction. Hence $M$ is Noetherian and the remaining implication is clear.

Corollary 3.3. Every Artinian submodule of a Max module is Noetherian.

Proof. The result is clear.

Notation. For $m \in M$ we equate $(m)$ with the cyclic submodule of $M$ generated by the element $m$.

LEMMA 3.4. For a module $M$ suppose that the sum $A / A_{1}+B / A_{1}$ is direct in $M / A_{1}$ where $A \supset A_{1}$ and $B \supset A_{1}$ Then the sum $A+B_{1} / B_{1}+$ 
$B / B_{1}$ is direct in $M / B_{1}$ whenever $B \supset B_{1} \supseteq A_{1}$. In particular, $A / A_{1}$ is embedded in $M / B_{1}$.

Proof. If $a+b=c \in B_{1}$ with $a \in A$ and $b \in B$, then $a=c-b \in$ $A \cap B$. The hypothesis implies that $(c)+A_{1}=(b)+A_{1} \subseteq B_{1}$. Hence, $b$ $\in B_{1}$ and the sum $A+B_{1} / B_{1}+B / B_{1}$ is direct. The lemma follows.

LEMMA 3.5. Consider the sequence of ordered pairs of submodules of $M$, $\left(A_{1}, S_{1}\right),\left(A_{2}, S_{2}\right), \ldots$ subject to $A_{n} \subset S_{n}$ and $S_{n} \cap A_{n+1}=A_{n}$ and $S_{n} \subset S_{n+1}$ for all $n \geq 1$. Let $A=\cup A_{i}$ for all $i \geq 1$. Then $S_{n} / A_{n}$ is embedded in $M / A$ for all $n \geq 1$. Furthermore, if a submodule $K$ of $M$ contains $A$ and the sum $K / A_{n}+S_{n} / A_{n}=M / A_{n}$ is direct then $S_{n}+A / A$ is a direct summand of $M / A$.

Proof. We claim $S_{n} \cap A=A_{n}$. From the hypothesis

Again

$$
S_{n} \cap A_{n+2}=S_{n} \cap A_{n+2}=S_{n} \cap A_{n+1}=A_{n} .
$$

$$
S_{n} \cap A_{n+3}=S_{n} \cap S_{n+2} \cap A_{n+3}=S_{n} \cap A_{n+2}=A_{n} \text {. }
$$

In like manner $S_{n} \cap A_{n+k}=A_{n}$ for all $k \geq 1$ and thus $S_{n} \cap A=\cup\left(S_{n} \cap\right.$ $\left.A_{i}\right)(i \geq 1)=A_{n}$. Therefore, $S_{n}+A / A \cong S_{n} / A_{n}$ and $S_{n} / A_{n}$ is embedded in $M / A$. The remaining part follows directly from Lemma 3.4.

If a minimal submodule $A$ of $M$ has zero intersection with the radical of $M$ then $A$ has zero intersection with some maximal submodule. Hence $A$ is a direct summand in $M$. This "direct summand" property characterizes elements not in the radical in the "sense of our next lemma."

LEMMA 3.6. An element $x$ does not belong to the radical of $M$ if and only if $(x) / B$ is a minimal submodule and is a direct summand in $M / B$ for some $B \subset(x)$.

Proof. Let $x \in M-\operatorname{rad} M$. Then $(x)+K=M$ for some maximal submodule $K$ in $M$. Let $B=(x) \cap K$ and $B$ is a maximal submodule in $(x)$. The sum $(x) / B+K / B$ is direct and equals $M / B$. The other implication is clear.

A module is said to be completely reducible if it is equal to its socle. If the sum $H+N=M$ of submodules $H$ and $N$ of $M$ is direct and $H$ is completely reducible then $H \cap \operatorname{rad} M=(0)$. This follows from the fact 
that the sum $\operatorname{rad} M=\operatorname{rad} H+\operatorname{rad} N$ is direct and $\operatorname{rad} H=(0)$. We use this fact below.

THEOREM 3.7. Assume that factor modules of $M$ are Max Modules. If $M$ is infinitely generated then some factor module has an infinitely generated socle and a zero radical.

Proof. Let $M$ be a Max module which is infinitely generated. We will construct a sequence which will satisfy the hypothesis of Lemma 3.5. By Lemma 3.6 there is $x \in M-\operatorname{rad} M$ such that the sum $(x) / A_{1}+K_{1} / A_{1}=$ $M / A_{1}$ is direct where $A_{1} \subset(x)$ and $A_{1} \subset K_{1}$ and $(x) / A_{1}$ is a minimal submodule. Equate $S_{1}=(x)$. We apply this argument to $\bar{K}_{1}=K_{1} / A_{1}$. Since $M$ is infinitely generated and $S_{1}$ is finitely generated and contains $A_{1}$, $\bar{K}_{1}$ is infinitely generated. Since $\bar{K}_{1}$ is Max module, $\operatorname{rad} \bar{K}_{1} \neq \bar{K}_{1}$. By Lemma 3.5 there is $\bar{y} \in \bar{K}_{1}-\operatorname{rad} \bar{K}_{1}$ such that the sum $(\bar{y}) / \bar{A}_{2}+\bar{K}_{2} / \bar{A}_{2}=$ $\bar{K}_{1} / \bar{A}_{2}$ is direct where $\bar{A}_{2} \subset(\bar{y})$ and $\bar{A}_{2} \subset \bar{K}_{2}$ and $(\bar{y}) / \bar{A}_{2}$ is a minimal submodule. Summing up by Lemma 3.4 the sum $(x)+A_{2} / A_{2}+(y) / A_{2}+$ $K_{2} / A_{2}=M / A_{2}$ is direct and the first two summands are minimal submodules. Equate $S_{2}=(x)+(y)$. Clearly $S_{1} \cap A_{2}=A_{1}$ because $K_{1} \cap$ $S_{1}=A_{1}$ and $S_{2} / A_{2}$ is a completely reducible module of length two. Also, $A_{2} \subset K_{1}$ and $A_{2} \subset K_{2}$. By this process we construct a sequence ( $a_{1}$, $\left.S_{1}, K_{1}\right),\left(A_{2}, S_{2}, K_{2}\right), \ldots$ subject to $A_{n} \subset S_{n}$ and $S_{n} \cap A_{n+1}=A_{n}$ and $S_{n} \subset$ $S_{n+1}$ and $S_{n} / A_{n}$ is a completely reducible factor module of length $n$ for all $n \geq 1$. Furthermore, $A_{n} \subset K_{i}$ for all $n \geq 1$ and all $i \geq 1$ and the sum $S_{n} / A_{n}+K_{n} / A_{n}=M / A_{n}$ is direct. Let $A=\cup A_{n}(n \geq 1)$. By Lemma 3.5 every completely reducible factor module $S_{n} / A_{n}$ of length $n$ is embedded in $M / A$. Let $S=\cup S_{i}(i \geq 1)$. Then $S / A$ is infinitely generated and belongs to the socle of $M / A$. Also each $S_{n}+A / A$ is a direct summand of $M / A$, recall the sum $S_{n} / A_{n}+K_{n} / A_{n}=M / A_{n}$ is direct, see Lemma 3.6. Therefore, from the remark preceding this theorem the completely reducible module $S_{n}+A / A$ has zero intersection with $\operatorname{rad}(M / A)$. Thus, $S / A$ misses $\operatorname{rad} M / A$ and is embedded in the factor module $(M / A) / \mathrm{rad}$ $(M / A)$ which has zero radical. This completes the proof.

THEOREM 3.8. The following conditions for a module $M$ are equivalent:

(1) The module $M$ is Noetherian.

(2) Every factor module of $M$ is a Max module and has a finitely generated socle.

(3) In a factor module of $M$, the radical of a submodule is small and the socle is finitely generated. 
(4) In a factor module the radical and the socle of a submodule are finitely generated.

Proof. Condition (3) as well as condition (4) implies that factor modules of $M$ are Max modules. The remaining implications follow from Theorem 3.7.

Definition 3.9. (Kurshan, [7] p. 376) A ring $R$ with 1 is called a T.C. ring if it has the following property: if $K$ is a submodule of a cyclic $R$-module and has a minimal essential socle, then $K$ is finitely generated. Equivalently, $R$ is T.C. if each cyclic $R$-module which has a finitely generated essential socle is Noetherian [7, p. 380].

Proposition 3.10. Let $M$ be a module with the property that if $K$ is a submodule of a factor module and has a minimal essential socle then $K$ is finitely generated. Then factor modules of $M$ are Max modules.

Proof. We argue by contradiction. Suppose $A$ is a nonzero submodule of $M$ which contains no maximal submodule. Let $x \in A-(0)$ and let $B$ be a maximal submodule of $(x)$. Let $E$ be maximal among the submodules $H$ subject to $x \in M-H$ and $A \supseteq H \supset B$. Clearly, $A / E$ has a minimal essential socle (namely $(x)+E / E$ ) and is infinitely generated otherwise $A$ would contain a maximal submodule. This contradicts the hypothesis and we conclude that $A=(0)$.

Corollary 3.11. (Kurshan [7]) A ring $R$ with 1 is Noetherian if and only if $R$ is a T.C. ring and the socle of every factor module of $R$ is finitely generated.

Proof. Since $R$ has 1 , a cyclic $R$-module is a factor module of $R$. Hence, $R$ is a Max module by Proposition 3.10, and is Noetherian by Theorem 3.9.

Kurshan's conditions extend to modules.

Corollary 3.12. (Kurshan) $A$ module is Noetherian if and only if every submodule of every factor module of $M$ which has a minimal essential socle is finitely generated and every factor module of $M$ has a finitely generated socle.

Proof. The proof follows from Proposition 3.10 and Theorem 3.8. 
Example 3.13. The converse of Proposition 3.10 is false. There exists a Max ring $R\left(R_{R}\right.$ is a Max module) with 1 which is not a T.C. ring. Let $R$ be the commutative ring generated by $1, p, x_{1}, y_{1}, x_{2}, y_{2} \ldots$ where all products are zero except $x_{1} y_{1}=p$ and $l z=z$ for all $z$ in $R$; also $z+z=0$ for all $z$ in $R$. The socle of $R$ is $\{0, p\}$ and is essential. The radical of $R$ is infinitely generated by $\left\{p, x_{1}, y_{1}, x_{2}, y_{2}, \ldots\right\}$. Therefore, $R$ is not Noetherian but factor modules of $R$ are Max modules.

4. The completely reducible module. Recall a module $M$ is completely reducible if $s(M)=M$. In such cases there is a well-ordered increasing (decreasing) sequence $A_{1} \subset A_{2} \subset \ldots\left(B_{1} \supset B_{2} \supset \ldots\right)$ such that $A_{n+1} / A_{n}$ is a minimal submodule $\left(B_{n+1}\right.$ is a maximal submodule of $\left.B_{n}\right)$. Clearly, the Min module (Max module) extends the increasing (decreasing) notion of the completely reducible module. We look for condition on a Min module $K$ for which $K / \operatorname{rad} K$ is a completely reducible module.

LEMMA 4.1. Suppose that $B$ is a direct summand of a module $M$ and the radical of $M$ is zero. Then $B+(x)$ is a direct summand of $M$ for every minimal submodule $(x)$ of $M$.

Proof. Assume the notation of the hypothesis, $M=B+H$ is direct for some appropriate submodule $H$ of $M$. Assume $(x) \cap B=(0)$. Then $x=$ $b+h$ with $b$ in $B$ and $h$ in $H$. Since $(x) \cap B=(0)$ it follows that $(x)=(x)$ $+(b) /(b) \cong(h)+(b) /(b) \cong(h)$. The $\operatorname{rad} M=\operatorname{rad} B+\operatorname{rad} H=(0)$ forces $\operatorname{rad} H=(0)$. Hence, $(h)$ is a minimal submodule of $H$ and $H=(h)+E$ where $E$ is some maximal submodule of $H$ and $h \in H-E$. The sum $B+$ $(x)+E=M$ is direct since $B+(x)=B+(h)$. This completes the proof.

Proposition 4.2. A module is completely reducible if and only if the socle is essential and the radical is zero and every cyclic submodule has a finitely generated socle.

Proof. For $b \in M$ we claim that $\operatorname{rad}(b)=(0)$ and let $y \in(b)-(0)$. Since $\operatorname{rad} M=(0)$ there is a maximal submodule $K$ of $M$ such that $y \in M$ $-K$. Hence $K \cap(b)$ is maximal in $(b)$ and $\operatorname{rad}(b)=(0)$. By the hypothesis the socle of $(b)$ is finitely generated. We use Lemma 4.1 applied to $(b)$ a finitely many times to conclude that the socle of $(b)$ is a direct summand of $(b), s((b))+E=(b)$ where $E$ is an appropriate submodule of $(b)$. Since the socle of $M$ is essential in $M$, the socle of $(b)$ is essential in $(b)$ and $E=(0)$. Therefore, $b \in s(M)$ which completes the proof. 
Corollary 4.3. Let $M$ be a Soc module in which every cyclic submodule of every factor module has a finitely generated socle. Then $M / \operatorname{rad} M$ is completely reducible. In particular, if $M$ is Artinian then $M / \operatorname{rad} M$ is completely reducible.

Proof. The proof follows directly from Proposition 4.2.

The author thanks the referee for his comments on $\$ 2$.

\section{REFERENCES}

1. J. Dieudonne, Les Ideaux Minimaux dans les Anneaux Associatifs, Proc. Int. Cong. Mathematicians, 2 (1950), 44-48.

2. S. Dickson, $A$ torsion theory for abelian categories, Trans. Amer. Math. Soc., 121 (1966), 223-235.

3. N. J. Divinsky, Ring and radicals, University of Toronto Press, 1965.

4. A. W. Goldie, Semiprime rings with the maximum condition, Proc. London Math. Soc., 10 (1960), 201-220.

5. N. Jacobson, Structure of Rings, American Mathematical Society Colloquium, Vol. 36. Rev. ed. Providence, R.I.: 1964.

6. R. E. Johnson, Extended centralizer of a ring over a module, Proc. Amer. Soc., 2 (1951), 891-895.

7 R. P. Kurshan, Rings whose cyclic modules have finitely generated socle, J. Algebra, 15 (1970, 376-386.

8. J. Lambek, Torsion Theories, Additive Semantics and Rings of Quotients, Vol. 177 Springer-Verlag, New York 1971.

9. __ Lectures on rings and modules, Blaisdell, Waltham, Mass. 1966.

10. H. Prufer, Untersuchungen uber die Zerlegbarkeit der abzahlbaren primaren abelschen Gruppen, Math. Z., 17 (1923), 35-61.

11. R. Shock, Certain Artinian rings are Noetherian, Canad. J. Math., 24 (1972), 553-556.

Received October 26, 1973 and in revised form April 24, 1974.

SOUTHERN ILLINOIS UNIVERSITY 



\section{PACIFIC JOURNAL OF MATHEMATICS}

\section{EDITORS}

RICHARD ARENS (Managing Editor)

University of California

Los Angeles, California, 90024

\section{R. A. BEAUMONT}

University of Washington

Seattle, Washington 98105

\section{J. DugunDJI}

Department of Mathematics University of Southern California Los Angeles, California 90007

D. Gillbarg AND J. MilgRAM

Stanford University Stanford, California 94305

\section{ASSOCIATE EDITORS}

E. F. BECKENBACH

B. H. NEUMANN

SUPPORTING

UNIVERSITY OF BRITISH COLUMBIA

UNIVERSITY OF CALIFORNIA

MONTANA STATE UNIVERSITY

UNIVERSITY OF NEVADA

NEW MEXICO STATE UNIVERSITY

OREGON STATE UNIVERSITY

UNIVERSITY OF OREGON OSAKA UNIVERSITY
F. WOLF

K. YosHIDA

INSTITUTIONS

UNIVERSITY OF SOUTHERN CALIFORNIA

STANFORD UNIVERSITY

UNIVERSITY OF TOKYO

UNIVERSITY OF UTAH

WASHINGTON STATE UNIVERSITY

UNIVERSITY OF WASHINGTON

AMERICAN MATHEMATICAL SOCIETY

NAVAL WEAPONS CENTER

The Supporting Institutions listed above contribute to the cost of publication of this Journal, but they are not owners or publishers and have no responsibility for its content or policies.

Mathematical papers intended for publication in the Pacific Journal of Mathematics should be in typed form or offset-reproduced, (not dittoed), double spaced with large margins. Underline Greek letters in red, German in green, and script in blue. The first paragraph or two must be capable of being used separately as a synopsis of the entire paper. Items of the bibliography should not be cited there unless absolutely necessary, in which case they must be identified by author and Journal, rather than by item number. Manuscripts, in duplicate if possible, may be sent to any one of the five editors. Please classify according to the scheme of Math. Rev. Index to Vol. ${ }^{39}$. All other communications to the editors should be addressed to the managing editor, or Elaine Barth, University of California, Los Angeles, California, 90024.

100 reprints are provided free for each article, only if page charges have been substantially paid. Additional copies may be obtained at cost in multiples of 50 .

The Pacific Journal of Mathematics is issued monthly as of January 1966. Regular subscription rate: $\$ 72.00$ a year (6 Vols., 12 issues). Special rate: $\$ 36.00$ a year to individual members of supporting institutions.

Subscriptions, orders for back numbers, and changes of address should be sent to Pacific Journal of Mathematics, 103 Highland Boulevard, Berkeley, California 90708.

\section{PUBLISHED BY PACIFIC JOURNAL OF MATHEMATICS, A NON-PROFIT CORPORATION}

Copyright (C) 1974 by Pacific Journal of Mathematics

Manufactured and first issued in the U.S.A. 


\section{Pacific Journal of Mathematics}

\section{Vol. 54, No. $2 \quad$ June, 1974}

John Edward Coury, Walsh series with coefficients tending monotonically to

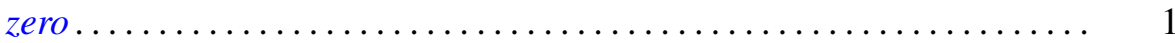

Patrick Michael Fitzpatrick and Walter Volodymyr Petryshyn, Fixed point theorems for multivalued noncompact acyclic mappings ............

Irving Leonard Glicksberg, More on Phragmén-Lindelöf for function

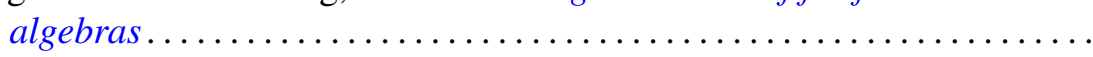

Adilson Goncalves, Structural constants. II .................. 39

Richard P. Gosselin, Closure theorems for affine transformation groups .... 53

Ralph Peter Grimaldi, Baer and UT-modules over domains ........... 59

Edward Grossman, On the prime ideal divisors of $\left(a^{n}-b^{n}\right) \ldots \ldots \ldots \ldots . \ldots 73$

A. Hedayat and Ester Seiden, On the theory and application of sum composition of Latin squares and orthogonal Latin squares.......... .

Gerald L. Itzkowitz, Continuous measures, Baire category, and uniform continuity in topological groups ......................... 115

Francis Masat, Right simple congruences on a semigroup ............ 127

Robert Harvey Oehmke, Right congruences and semisimplicity for Rees matrix semigroups..................................

Qazi Ibadur Rahman and Jan Stankiewicz, Differential inequalities and local valency . . . . . . . . . . . . . . . . . . . . . . . . . . . . . . . . . . . . . 165

William John Reed, Random points in a simplex ................ 183

Mohan S. Shrikhande, Strongly regular graphs and group divisible

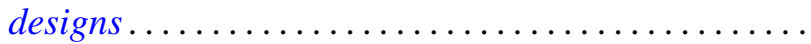

Zahava Shmuely, The structure of Galois connections ... . .

Robert C. Shock, Dual generalizations of the Artinian and Noetherian

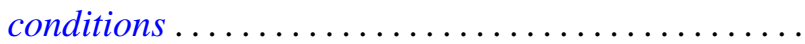

Arne Stray, Approximation and interpolation for some spaces of analytic functions in the unit disc ........................

Eldon Jon Vought, Monotone decompositions into trees of Hausdorff continua irreducible about a finite subset ............

James Wirth, The mapping cylinder axiom for WCHP fibrations ... 263

Gordon S. Woodward, Invariant means and ergodic sets in Fourier analysis... 